\title{
The shuttle walking test: a reproducible method for evaluating the impact of shortness of breath on functional capacity in patients with advanced cancer
}

\author{
S Booth ${ }^{\star}$, L Adams
}

\begin{abstract}
Background-Breathlessness leading to exercise limitation is common in patients with advanced cancer and is ineffectively treated. There are few research data to guide clinicians on best practice. The shuttle walking test has been validated for some conditions such as chronic obstructive pulmonary disease but not for advanced cancer. One of the well documented difficulties of doing clinical research in palliative care is the acceptability of assessment tests. This study examined the reproducibility of the shuttle walking test in patients with advanced cancer to help facilitate the systematic evaluation of interventions designed to improve breathlessness.

Methods-Patients performed three shuttle walks on separate days with continuous monitoring of arterial oxygen saturation and heart rate; simple pulmonary function $\left(F_{E V}\right)$ was also recorded. Data on quality of life, anxiety, and depression were collected throughout the study period using appropriate questionnaires. Breathlessness was measured before and after exercise using a visual analogue scale.

Results-Data from 22 patients were compared between visits 2 and 3 . There were no significant differences between the $\mathrm{FEV}_{1}(1.89 v 1.90, \mathrm{p}=0.73)$, distance walked on each test $(245 \mathrm{~m} v 256 \mathrm{~m}, \mathrm{p}=0.14)$, endexercise levels of heart rate $(107 / \mathrm{min} v$ $108 / \mathrm{min}, \quad p=0.11)$, oxygen saturation $(93.4 \% v 93.2 \%, p=0.38)$, or breathlessness scores $(p=0.62)$ on the two occasions. Indices of quality of life, anxiety, and depression were also not different between the two tests. The investigation was very acceptable to patients, families, and staff. Conclusions-The shuttle walking test is a reproducible test of functional capacity in ambulant patients with advanced cancer, WHO performance status 1 or 2 . The data indicate that a practice session is needed. It is easy to carry out and acceptable for patients with advanced cancer.

(Thorax 2001;56:146-150)
\end{abstract}

Keywords: exercise test; shuttle walking test; breathlessness; advanced cancer

The breathlessness that frequently accompanies advanced cancer ${ }^{1}$ is a disabling symptom leading to exercise intolerance and is distressing for patients, their families, and the clinicians caring for them. Once cause directed treatments have been exhausted, there are no commonly effective treatment strategies for symptom relief, ${ }^{2}$ in contrast with the use of analgesics for pain. An important difficulty in researching methods to relieve breathlessness is that there is no measure of the impact of breathlessness on functional capacity that has been validated for this group of patients. Breathlessness is the subjective experience of "that feeling associated with an uncomfortable need to breathe". ${ }^{3}$ Since there are no clinical signs that can be used to assess breathlessness reliably, investigators have relied on determining exercise capacity as an objective index of the severity of this symptom. The aim of this study was to investigate whether the shuttle walking test (SWT), ${ }^{4}$ first used for patients with chronic obstructive pulmonary disease (COPD) and now validated for patients with pacemakers ${ }^{5}$ and heart failure, ${ }^{6}$ is a reproducible test for patients with advanced cancer. Validation, if possible, would allow the investigation of different interventions for breathlessness and comparisons of efficacy.

In order to evaluate the reproducibility of the SWT, it was important to establish that neither the clinical nor the psychological status nor the quality of life of the patients participating in the study was significantly different on each of the occasions on which the test was performed. We therefore measured indices of the physiological response to exercise, simple pulmonary function, and indicators of quality of life, anxiety, and depression since any changes in these factors could have a confounding influence on exercise performance.

\section{Methods}

The shuttle walking test was developed for use in patients from a fitness test used in athletes ${ }^{47}$ and has been validated in comparison with the more frequently used treadmill exercise test. ${ }^{8}$ The methods used for performing the shuttle walking test followed those described by Singh and co-workers. ${ }^{4}$ The fact that this test is externally paced overcomes the objections that have made the self-paced walking tests ${ }^{9}$ lose favour as objective measures of functional capacity. ${ }^{10}$

With ethical approval, patients were recruited from the day centre and later from the ward and outpatients of an NHS hospice. All the patients had cancer that was no longer curable and were receiving palliative care which included chemotherapy, hormonal treatment, or radiotherapy where indicated. The patients were all of WHO performance status 1 or 2 . Any patient with advanced cancer who could 
Table 1 Data from patients who completed three shuttle walking tests who were excluded from the study

\begin{tabular}{rrrrl}
\hline & \multicolumn{2}{l}{ Distance walked $(\mathrm{m})$} & \\
\cline { 2 - 4 } Patient no & Test 1 & Test 2 & Test 3 & Reasons for exclusion \\
\hline 8 & 80 & 90 & 120 & Not SOB, back pain \\
10 & 140 & 140 & 210 & Transfused between tests 2 and 3 \\
12 & 100 & 130 & 120 & Not SOB, giddy \\
13 & 170 & 150 & 170 & Not SOB, leg pain \\
14 & 150 & 70 & 70 & Chest infection between tests 2 and 3 \\
16 & 20 & 20 & 20 & Not SOB, lack of mobility \\
17 & 270 & 300 & 270 & Not SOB, ankle pain \\
19 & 80 & 70 & 60 & Developed pleural effusion between tests 2 and 3 \\
27 & 140 & 150 & 210 & Baseline SOB very different on tests 2 and 3 \\
30 & 200 & 260 & 350 & Not SOB, knee hurt \\
\hline
\end{tabular}

SOB $=$ short of breath.

walk without help from another person, even if using a walking aid, was eligible to take part. Patients with cognitive impairment, short term memory loss, or difficulty in walking because of pain were excluded. After informed consent had been obtained, in accordance with the guidelines laid down by the Royal College of Physicians, ${ }^{11}$ patients were asked to fill in the European Organisation for Research and Treatment of Cancer questionnaires (QLQC30, LC13 $)^{12} 13$ and the Hospital Anxiety and Depression scale (HAD) ${ }^{14}$ They were always asked to rate their general level of breathlessness on the modified MRC scale. ${ }^{15}$ Help with questionnaires was given if required.

Patients were taken to the beginning of the course which was set up in an unobstructed and quiet corridor maintained at a constant temperature. Before starting, the procedure was explained in a standardised way and the patient was asked to rate their present level of breathlessness on a vertical visual analogue scale (VAS) ${ }^{16}$ Forced expiratory volume in one second $\left(\mathrm{FEV}_{1}\right)$ and forced vital capacity (FVC) were recorded three times using a portable spirometer (Model 3000, Microlab, Rochester, Kent UK). A pulse oximeter designed for ambulatory use (Model 5, Minolta, Japan) was then attached and baseline readings of heart rate and arterial oxygen saturation $\left(\mathrm{SaO}_{2}\right)$ were recorded. Patients were accompanied around the course if they wanted this and were encouraged to continue walking at the speed set by the tape until they had to stop. The same phrases were used by all investigators: these included "That's good, keep going as long as you can" and/or "You're doing fine, don't forget you have to go a bit faster now the beep has gone". Once seated, patients immediately rated their breathlessness on the VAS. They were then asked the reasons for stopping or being unable to keep up with the required pace using a standardised statement and question: "You had to stop, what was it that made you stop the test?" The patients' comments were recorded verbatim. Levels of $\mathrm{SaO}_{2}$ and heart rate at the end of the exercise were recorded.

The SWT was performed on three separate days. The data from the first familiarisation test were not analysed. The median interval between tests 2 and 3, which formed the basis of this study, was seven days (range 1-35 days). Thirty of the 71 patients refused to take part in the study after being recommended from the ward or day centre staff; 16 felt it was impractical to be brought into the day centre after discharge frequently enough to take part as they lived too far away, eight felt too frail either psychologically or physically to take on the mental as well as they physical aspects of the test, five felt that mobility was limited by pain or giddiness and it was agreed that it would be unhelpful as they would be excluded from the analysis, and one patient refused because her counsellor had set her the task of saying "No" to something that week.

\section{DATA ANALYSIS}

The descriptive statistical method described by Bland and Altman ${ }^{17}$ was used to present the quantitative data relating to the SWT and to the index of lung function. The HAD scale

Table 2 Quantitative data from shuttle walking tests

\begin{tabular}{|c|c|c|c|c|c|c|c|c|}
\hline \multirow[b]{2}{*}{ Patient no } & \multicolumn{3}{|c|}{ Distance walked ( $m$ ) } & \multirow[b]{2}{*}{$\begin{array}{l}\text { Interval between tests } 2 \text { and } 3 \\
\text { (days) }\end{array}$} & \multicolumn{2}{|c|}{ Heart rate } & \multicolumn{2}{|c|}{ Final VAS score } \\
\hline & Test 1 & Test 2 & Test 3 & & Test 2 & Test 3 & Test 2 & Test 3 \\
\hline 1 & 380 & 380 & 350 & 8 & 101 & 98 & 73 & 81 \\
\hline 2 & 190 & 180 & 160 & 12 & 97 & 106 & 69 & 73 \\
\hline 3 & 100 & 240 & 270 & 7 & 106 & 105 & 63 & 78 \\
\hline 4 & 60 & 90 & 90 & 7 & 84 & 82 & 74 & 71 \\
\hline 5 & 240 & 330 & 420 & 7 & 128 & 136 & 58 & 58 \\
\hline 6 & 120 & 120 & 130 & 7 & 84 & 91 & 49 & 69 \\
\hline 7 & 210 & 230 & 220 & 14 & 77 & 80 & 42 & 59 \\
\hline 9 & 180 & 260 & 260 & 7 & 125 & 129 & 19 & 27 \\
\hline 11 & 220 & 280 & 270 & 13 & 86 & 86 & 45 & 85 \\
\hline 15 & 280 & 360 & 300 & 14 & 103 & 107 & 19 & 11 \\
\hline 18 & 130 & 160 & 170 & 2 & 100 & 99 & 14 & 18 \\
\hline 20 & 220 & 170 & 270 & 6 & 104 & 104 & 70 & 80 \\
\hline 21 & 350 & 440 & 450 & 35 & NS & NS & 37 & 20 \\
\hline 22 & 240 & 340 & 380 & 5 & 148 & 141 & 21 & 16 \\
\hline 23 & 80 & 70 & 90 & 7 & 103 & 104 & 91 & 80 \\
\hline 24 & 610 & 570 & 570 & 4 & 128 & 129 & 99 & 99 \\
\hline 25 & 80 & 130 & 130 & 4 & 103 & 104 & 82 & 80 \\
\hline 26 & 150 & 200 & 220 & 1 & 108 & 110 & 87 & 90 \\
\hline 28 & 70 & 80 & 90 & 7 & 110 & 107 & 58 & 24 \\
\hline 29 & 120 & 150 & 160 & 7 & NS & NS & 71 & 42 \\
\hline 31 & 190 & 200 & 180 & 5 & 121 & 121 & 32 & 32 \\
\hline 32 & 450 & 400 & 460 & 7 & 118 & 127 & 67 & 86 \\
\hline Mean (SD) & $212(1.36)$ & $245(131)$ & $256(135)$ & & 106.7 & 108.3 & 56.4 & 58.1 \\
\hline $\mathrm{p}$ value ( $t$ test) & & 0.01 & 0.14 & & \multicolumn{2}{|c|}{0.11} & \multicolumn{2}{|c|}{0.62} \\
\hline Range & & $60-610$ & & $1-35$ & \multicolumn{2}{|c|}{$77-148$} & \multicolumn{2}{|c|}{$11-99$} \\
\hline
\end{tabular}

VAS = visual analogue scale. 
Table 3 Qualitative data from shuttle walking tests

\begin{tabular}{|c|c|c|c|c|c|c|c|c|}
\hline \multirow[b]{2}{*}{ Patient no } & \multicolumn{2}{|c|}{$M R C$ value } & \multicolumn{2}{|c|}{$H A D$ anxiety score } & \multicolumn{2}{|c|}{$H A D$ depression score } & \multicolumn{2}{|c|}{ EORTC score } \\
\hline & Test 2 & Test 3 & Test 2 & Test 3 & Test 2 & Test 3 & Test 2 & Test 3 \\
\hline 1 & 1 & 2 & 5 & 4 & 10 & 5 & 58.3 & 66.7 \\
\hline 2 & 1 & 3 & 14 & 13 & 9 & 11 & 50 & 41.7 \\
\hline 3 & 0 & 1 & 0 & 0 & 2 & 3 & 66.7 & 75 \\
\hline 4 & 1 & 1 & 0 & 0 & 3 & 2 & 83.3 & 75 \\
\hline 5 & 0 & 1 & 0 & 1 & 4 & 6 & 75 & 50 \\
\hline 6 & 1 & 2 & 12 & 14 & 7 & 5 & 100 & 66.7 \\
\hline 7 & 3 & 3 & 7 & 5 & 9 & 9 & 58.3 & 50 \\
\hline 9 & 2 & 1 & 7 & 7 & 5 & 5 & 66.7 & 66.7 \\
\hline 11 & 2 & 3 & 9 & 7 & 2 & 6 & 66.7 & 66.7 \\
\hline 15 & 0 & 0 & 2 & 2 & 4 & 3 & 66.7 & 83.3 \\
\hline 18 & 0 & 0 & 8 & 10 & 9 & 10 & 50 & 41.7 \\
\hline 20 & 2 & 2 & 17 & 15 & 17 & 20 & 0 & 25 \\
\hline 21 & 0 & 1 & 8 & 13 & 5 & 6 & 83.3 & 91.6 \\
\hline 22 & 1 & 0 & 0 & 0 & 2 & 1 & 83.3 & 75 \\
\hline 23 & 3 & 3 & 0 & 3 & 3 & 3 & 41.7 & 41.7 \\
\hline 24 & 0 & 0 & 0 & 0 & 0 & 0 & 100 & 50 \\
\hline 25 & 3 & 3 & 7 & 5 & 9 & 12 & 58.3 & 66.7 \\
\hline 26 & 4 & 4 & 12 & 12 & 16 & 16 & 50 & 50 \\
\hline 28 & 1 & 1 & 10 & 10 & 10 & 12 & 50 & 50 \\
\hline 29 & 1 & 2 & 9 & 7 & 6 & 5 & 66.7 & 66.7 \\
\hline 31 & 0 & 0 & 7 & 7 & 6 & 6 & 50 & 50 \\
\hline 32 & 2 & 1 & 7 & 5 & 5 & 3 & 50 & 66.7 \\
\hline Median & 1.0 & 1.0 & 7.0 & 6.0 & 5.5 & 5.5 & 62.5 & 66.7 \\
\hline $\mathrm{p}$ value $e^{\star}$ & & $>0.05$ & & $>0.05$ & & $>0.05$ & & $>0.05$ \\
\hline Range & & $0-4$ & & $0-17$ & & $0-20$ & & $0-100$ \\
\hline
\end{tabular}

*Wilcoxon signed rank test.

MRC = modified Medical Research Council dyspnoea scale; HAD = Hospital Anxiety and Depression scale; EORTC = European Organisation for Research Trials and Cancer.

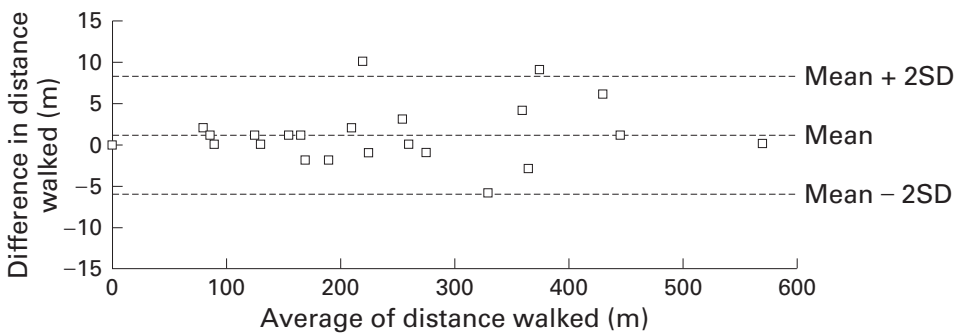

Figure 1 Relationship between the mean distance walked and the difference in the distance walked in metres.

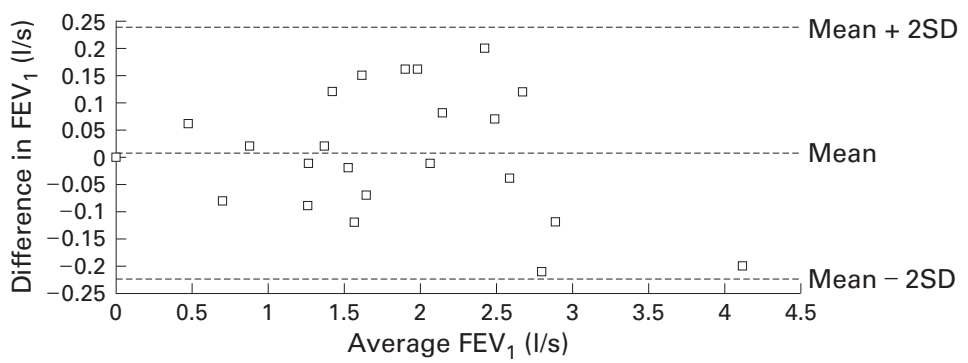

Figure 2 Relationship between the mean forced expiratory volume in one second (FEV $)$ and the difference in $F E V_{1}$ in $l / s$.

generates scores of $0-21$ and anxiety and depression are scored separately, with scores of over 11 indicating that further clinical assessment is needed. The EORTC questionnaires give scores of 0-100 indicating poor and good quality of life, respectively. The mean levels across the two SWTs were compared using paired $t$ statistics for quantitative data and a Wilcoxon signed rank test for questionnaire derived data. A p value of $<0.05$ in a two tailed test was taken to indicate a statistically significant difference for any variable.

Results

Of the 41 patients entering the study, nine who did at least one SWT could not complete the protocol. Eight had deteriorated significantly by the time the next walk was planned; most were too weak to get out of bed and walk. One patient, however, did not enjoy his first walking test and so declined further involvement. Of the 32 patients completing the three tests, the data from 10 patients were excluded from further analysis. The reasons for their exclusion are shown in table 1 .

The median age of the 21 patients (nine men) included in the analysis was 70 years (range 31-84); three patients had carcinoma of the lung, five had metastatic breast cancer (two of whom had pleural effusions), and the remaining patients had various other primary cancers. Eighteen patients were recruited from the hospice day centre and one each from the ward, outpatients, and the community. The average percentage predicted $\mathrm{FEV}_{1} / \mathrm{FVC}$ ratio for the population studied was 95\% (range 65-120\%).

Table 2 shows the distance walked in metres by each patient on each occasion together with the quantitative data on heart rate and final VAS value. The interval between visits 2 and 3 (in days) is also shown. The qualitative questionnaire derived data on breathlessness, quality of life, anxiety, and depression are shown in table 3 . There were no statistically significant differences between any of these measures on the two occasions.

The difference in the distance walked by the participants (in metres) between the two tests with reference to the mean distance walked is shown in fig 1. Although on average patients walked further on the second test, this difference was small and not statistically significant $(p=0.14)$. Figure 2 shows the $F_{E V}$ data plotted in the same way. There was no significant difference for this index of lung function across the two test days $(p=0.73)$. Figure 3 shows the data for peak heart rate; the lack of a significant difference $(p=0.11)$ suggests that the two tests were performed with 


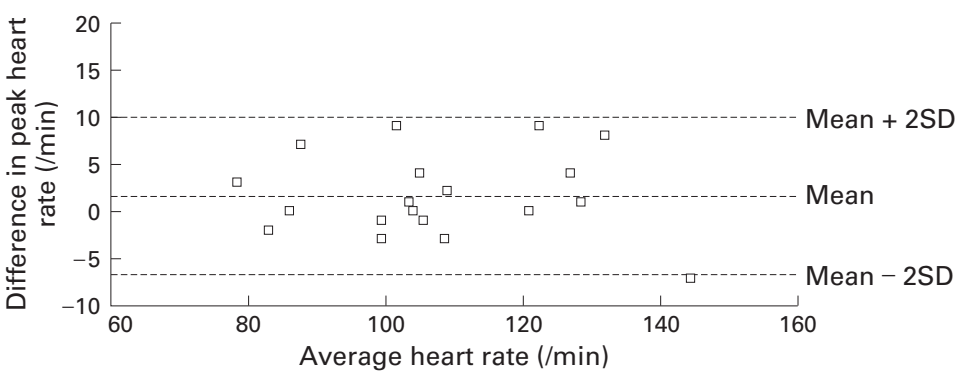

Figure 3 Relationship between mean final heart rate and difference in final heart rate.

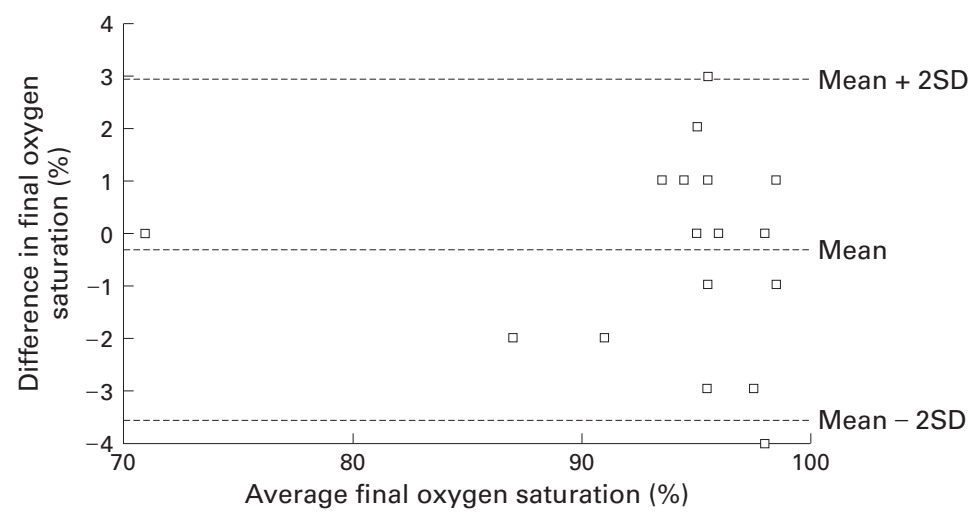

Figure 4 Relationship between mean final arterial oxygen saturation and difference between final oxygen saturation.

equivalent degrees of physical exertion. Figure 4 shows the results for final arterial oxygen saturation; this did not differ between the two tests $(p=0.38)$, indicating that exercise related hypoxaemia was not a confounding factor. Figures 1-4 show the mean difference and 95\% confidence interval ( -2 SD to +2 SD of difference) between visits 2 and 3 .

\section{Discussion}

The main finding from this study is that the SWT is a reliable and reproducible test of the impact of breathlessness on functional capacity in patients with advanced cancer who are mobile and whose clinical condition is stable. Patients will exercise to their maximum capacity, limited by breathlessness, on each occasion that the test is performed. The peak heart rate values and final VAS scores demonstrate this. The external pacing provided by the audiotape helps the patient to exercise maximally.

Although there was a large range in the distance walked $(60-570 \mathrm{~m})$, the mean difference between tests was only $1.40 \mathrm{~m}$. The stability of the $\mathrm{FEV}_{1}$ measurements $(\mathrm{p}=0.73)$ shows that the patients were clinically stable during this time and the data for final oxygen saturation also showed this to be a consistent factor. The mean percentage predicted $\mathrm{FEV}_{1} / \mathrm{FVC}$ ratio for the population studied was $95 \%$ (range $65-$ $120 \%$ ) which shows that the study population did not have a higher than average incidence of COPD which could have biased our data and influenced our interpretation.

All the patients in this study reported that breathlessness was the major factor in stopping the test. The qualitative data show that the psychological status of the patients, their quality of life, and their perception of their general level of dyspnoea remained constant throughout the study period.

There is a natural hesitation in asking patients with advanced disease and a short prognosis to take part in research. Seventy one people were actually invited to take part and only 32 completed the protocol. As the study progressed we were better able to select those patients recommended to us who were likely to be able to enjoy the physical and mental demands of the shuttle test and who were going to be capable of completing it before asking them to take part. Those patients who had been admitted to the hospice after a recent physical deterioration were likely to feel too unwell even to contemplate participating. Those who had recently had successful symptom control often felt stimulated by the challenge and were heartened by the demonstration of their physical capabilities. Any patients with intractable family or psychological difficulties were unlikely to be able to face it, even as an idea. The test is not usually suitable for patients with cancer of WHO performance status 3 or 4 as they are likely to be in the phase of their illness where rapid deterioration and death is possible. Most of the patients who completed the three tests were recruited from the day centre or outpatients department which they were attending regularly and were living at home. In addition, 16 of the patients who were counted as refusals were not attending the day centre regularly and would have had to be brought in especially from some distance away. Patients with advanced cancer attend many hospital appointments and each is prolonged, so it was not possible to combine, for example, an outpatient appointment or radiotherapy treatment and an SWT on the same day. Consequently, our records of their comments show that many would have been willing to do the test but it was not practical. If the test became part of clinical assessment and is of possible direct benefit for an individual, it would be reasonable to make a specific appointment for it. This is not the case when asking patients to take part in a study where the results would be likely to benefit only other people. Six of the 10 patients excluded after completing three shuttle tests never became breathless. Their functional capacity was limited by other factors such as pain or "dizziness". We were investigating the SWT as a reproducible measure of the impact of breathlessness on functional capacity and so patients who never became breathless were excluded. The other four patients experienced a significant change in their clinical condition that would have affected the study of reproducibility of the test.

The SWT is an easily performed test that needs little equipment and minimal staff training for it to be completed accurately. It can be carried out in any clinical unit without the need for special facilities and takes relatively little time to complete. Walking is an everyday activity, which makes this test likely to be more acceptable to both the staff and the patients of a hospice than treadmill exercise testing. Its familiarity also allows patients to relate their 
abilities in the supervised exercise of the SWT to their normal life and the majority of patients performing the test reported an increased confidence in their physical abilities that was carried into their everyday activities.

We were very concerned about carrying out an exercise test in this group of patients. It is unusual to stress physically patients with advanced cancer; we were greatly encouraged by the positive effects that the study itself seemed to have on many of the patients taking part. It was not unusual for patients to be apprehensive before the first shuttle test but to volunteer favourable comments after the first and to look forward to the others. The research team did not seek comments but the day centre staff reported that many of the participants had found it an encouraging experience because they had not realised that they were capable of achieving so much physically. It had also increased the patients' confidence and that of the relatives in what they were able to do-for example, some patients who had used a wheelchair to get from the car park to the day centre chose to walk this distance while they could. The reversal of deconditioning as a contribution to improving breathlessness in COPD is an important part of pulmonary rehabilitation. ${ }^{18}$ The evidence for the general benefits of exercise is becoming clear from many branches of medicine $\mathrm{e}^{1920}$ and should not be neglected, tailored for each individual, for patients with advanced cancer. Most patients suitable for the SWT will be of performance status 1 or 2 .

It has been increasingly recognised that breathlessness is a common, frequently intractable symptom in patients with advanced cancer. The importance of non-pharmacological strategies for managing breathlessness has been underlined recently ${ }^{21}$ and these are of benefit for patients with breathlessness of all degrees of severity, whether physically active or not. The area of greatest therapeutic uncertainty at present lies with the treatment of patients who are breathless solely on exercise. The efficacy of the drugs used to treat breathlessness is still in question, ${ }^{22}$ particularly in the ambulant patient, and one of the difficulties of research is the lack of a standardised test of the impact of breathlessness on functional capacity for this patient group. The SWT should be ideal for testing these treatments in this group of breathless patients.

A major concern for clinical research in palliative medicine is the rapid rate of deterioration of the patients so that intervention studies are often difficult to complete. Four patients had to be excluded from the study because their clinical condition changed markedly such that reproducibility of the shuttle test was not possible to evaluate. Another difficulty with research in this group of patients is related to the fact that, because cancer is a multisystem disorder, there are many other potential problems that may limit mobility including pain or general weakness. We excluded any patients whose primary reason for stopping the test was unrelated to breathlessness (see table 1) and this was decided by the patient in response to our standard question when the test had been completed. This was because we were solely interested in testing the reproducibility of the SWT as a method of evaluating the impact of breathlessness on an individual's functional capacity. Cancer is also a disease of advancing age and concurrent nonmalignant conditions may also limit functional capacity. The SWT will not be suitable for all patients with breathlessness and advanced cancer but does provide a research tool for ambulant patients with breathlessness where intervention studies are needed. It may be useful as a clinical intervention in designing exercise programmes for rehabilitation in this group of people.

Dr Booth was supported by a Health Service Research Training Fellowship. Research nurse time and equipment was financed by a grant from Oxford District Health Services Research Committee. The Breathlessness Research Charitable Trust gave additional support. The authors would like to thank Rosemary Endsor and Frances Chamberlain for their help with data collection and their interest in the study, and Ann Green and Gill McEvedy for facilitating the work in the day centre. We would also like to thank Dr Nick Cox for extensive help in preparation of the manuscript and Dr R Twycross, Dr J Morris, Dr R J O Davies, and Dr D Lane of Sir Michael Sobell House and the Osler Chest Unit for advice, comment, and permission to approach their patients.

1 Reuben DB, Mor V. Dyspnea in terminally ill cancer patients. Chest 1986;89:234-6.

2 Higginson I, McCarthy M. Measuring symptoms in terminal cancer: are pain and dyspnoea controlled? $\mathcal{F} R$ Soc Med 1989;82:264-7.

3 Adams L, Chronos N, Lane R, et al. The measurement of breathlessness induced in normal subjects, validity of two scaling techniques. Clin Sci 1985;69:7-16.

4 Singh SJ, Morgan MDL, Scott S, et al. The development of Singh SJ, Morgan MDL, Scott S, et al. The development of
the shuttle walking test of disability in patients with chronic the shuttle walking test of disability in patient

5 Payne GE, Skehan JD. Shuttle walking test: a new approach for evaluating patients with pacemakers. Heart 1995;75: 414-8.

6 Kell SD, Chambers JS, Francis DP, et al. Shuttle-walk test to assess chronic heart failure. Lancet 1998;352:705.

7 Leger LA, Lambert JA. A maximal multistage 20-m shuttle shuttle run test to predict $\mathrm{VO}_{2}$ max. Eur $\mathcal{F}$ Appl Physiol 1982;49:1-12.

8 Singh SL, Morgan MDL, Hardman AE, et al. Comparison of oxygen uptake during a conventional treadmill test and the shuttle walking test in chronic airflow limitation. Eur Respir f1994;7:2016-20.

9 Butland RJA, Pang, J, Gross ER, et al. Two, six and 12 minute walking tests in respiratory disease. BMF 1982;284: minute

10 Guyatt GH, Pugsley SO, Sullivan MJ, et al. Effect of encouragement on walking test performance. Thorax 1984; 39:818-22.

11 Royal College of Physicians. Research involving patients. London: Royal College of Physicians, 1990.

12 Aaronson NK, Ahmedzai S, Bergman B, et al. The European Organisation for Research and Treatment of Cancer QLQ-C30: a quality of life instrument for use in international clinical trials in oncology. $\mathcal{F}$ Natl Cancer Inst 1993;85:365-76.

3 Bergman B, Aaronson NK, Ahmedzai S, et al. The EORTC QLQ-LC13: a modular supplement to the EORTC core Quality of Life Questionnaires (QLQ-C30) for use in lung cancer clinical trials. Eur f Cancer 1994;30A:635-42.

14 Zigmund AS, Snaith RP. The hospital anxiety and depression scale. Acta Psychiatry Scand 1983;67:361-70.

15 Mahler D, Wells CK. Evaluation of clinical methods for rating dyspnoea. Chest 1988;93:580-6.

16 Gift AG. Validation of a vertical visual analogue scales a measure of clinical dyspnea. Rehab Nurs 1989;14:323-5.

17 Bland JM, Altman DG. Statistical methods for assessing agreement between two methods of clinical measurement. agreement between two

18 Niederman MS, Clemente PH, Fein AM, et al. Benefits of a multidisciplinary pulmonary rehabilitation program: improvements are independent of lung function. Chest 1991; 99:798-804

19 Heinnonen A, Kannus P, Sievanan H, et al. Randomised controlled trial of effect of high impact exercise on selected risk factors for osteoporotic fractures. Lancet 1996;346: 1343-7.

20 Serres I, Varray A, Vallet G. Improved skeletal muscle performance after individualised exercise training in patients with chronic obstructive pulmonary disease. $\mathcal{F}$ Cardiopulm-Rehabil 1997;17:232-8.

21 Corner J, Plant H, Hern A, et al. Non-pharmacological intervention for breathlessness in lung cancer. Palliative Med 1996;10:299-305.

22 Davis CL. The therapeutics of dyspnoea. In: cancer surveys: problem areas in pain and symptom management. London: Imperial Cancer Research Fund, 1994;21:85-98. 